\title{
Strategies for the Chemoenzymatic Synthesis of \\ Deoxysugar Nucleotides: Substrate Binding Versus
}

Catalysis

Kwang-Seuk Ko, Corbin J. Zea, and Nicola L. Pohl*

Department of Chemistry and the Plant Sciences Institute, Gilman Hall, Iowa State University, Ames, Iowa 50011-3111

\section{Contents:}

Michaelis-Menten plots

General materials and methods

Details of mass spectrometry analysis with calibration curves 


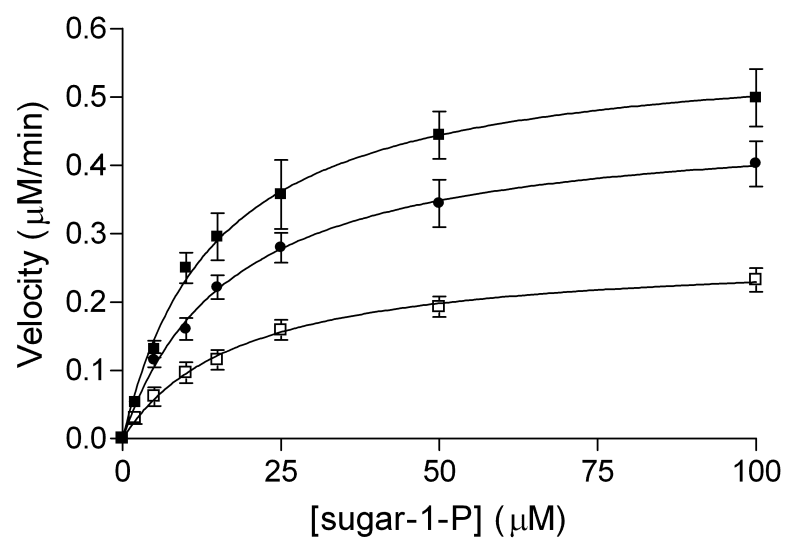

FIGURE S1. Michealis-Menten plots in the direction of UDP-glucose synthesis for E. coli UDP-glucose pyrophosphorylase and UTP in the presence of 6dGlc-1-phosphate (square), 4dGlc-1-phosphate (circle), and 3dGlc-1-phosphate (open square).

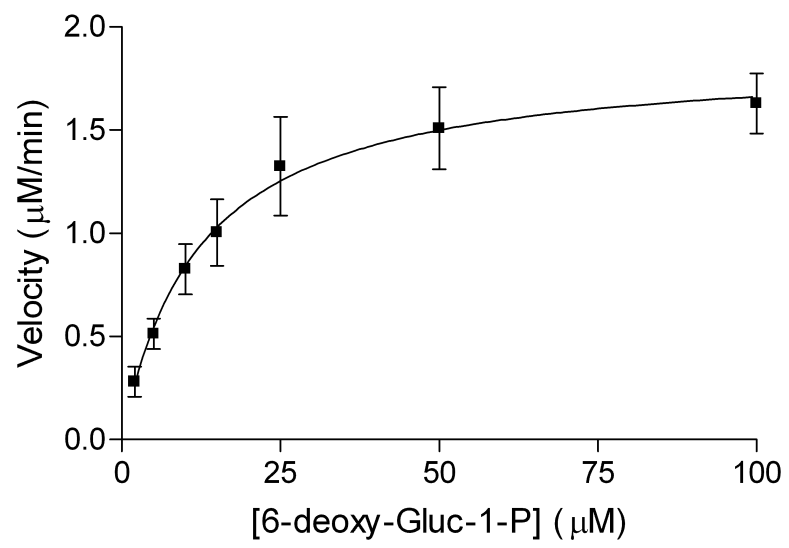

FIGURE S2. Michealis-Menten plot in the direction of UDP-glucose synthesis for yeast UDP-glucose pyrophosphorylase and UTP in the presence of 6dGlc-1-phosphate. 
General materials and methods. Trizma base and trizma hydrochloride were purchased from Fischer Scientific Company (Hanover Park, IL). Thermostable inorganic pyrophophatase (IPP) from Thermococcus litoralis (EC 3.6.1.1, M0296S) was purchased from New England Biolabs. All other chemicals were obtained from Sigma Chemical Co. (St. Louis, MO) unless otherwise noted and used without further purification. Yeast UDP-glucose pyrophosphorylase (EC 2.7.7.9, 9026-22-6) was desalted by buffer exchange using Microcon Centrifugal Filter Device, MWCO 10 kDa (Millipore, Billerica, MA). E. coli UDP-glucose pyrophosphorylase was prepared as previously reported. ${ }^{1}$ Nanopure water $(18.1 \mathrm{MHz})$ prepared from a Barnstead E-pure water purification system was employed throughout. Compounds 3-6 were synthesized and ${ }^{1} \mathrm{H}$ NMR, ${ }^{13} \mathrm{C}$ NMR, and ${ }^{31} \mathrm{P}$ NMR spectra match those previously reported. ${ }^{2}$

MS Conditions. A Shimadzu LCMS 2010 single quadrupole mass spectrometer (Shimadzu Scientific Instruments, Columbia, MD) equipped with an electrospray ionization (ESI) source was used in negative ion mode. The capillary temperature and the spray voltage were kept at $220{ }^{\circ} \mathrm{C}$ and $4.5 \mathrm{kV}$, respectively. The instrument was calibrated as previously reported ${ }^{1}$ by direct infusion of polyethylene glycol (PEG) 200, 600, $1000(1.5 \mu \mathrm{L} / \mathrm{L}, 2 \mu \mathrm{L} / \mathrm{L}$, and $15 \mu \mathrm{L} / \mathrm{L}$, respectively) and raffinose $(50 \mathrm{mg} / \mathrm{L})$ in water/methanol $(1: 1, \mathrm{v}: \mathrm{v})$ containing ammonium acetate $(0.19 \mathrm{mM}), 0.1 \%$ formic acid, and $0.1 \%$ acetonitrile. For sample analysis the solvent (acetonitrile/water/triethylamine, $35 / 65 / 0.2$ ) was constantly infused into the ion source at $250 \mu \mathrm{L} / \mathrm{min}$ by the attached Shimadzu HPLC pump and the samples were injected via the autosampler adapted to fit two 96-well plates. A preliminary MS chromatogram was obtained by scanning from 50 $-700 \mathrm{~m} / \mathrm{z}$. To increase the signal to noise ratio, the instrument was set for selected ion monitoring (SIM) mode and all relevant $\mathrm{m} / \mathrm{z}$ ions were monitored for further analysis of the enzymatic reactions. Inclusion of an Agilent Extend C18 column $(2.1$ x $50 \mathrm{~mm}$, Agilent, Palo Alto, CA) in the system further increased the signal to noise ratio and decreased the appearance of sodium ion adducts by $10-15 \%$ without separation of reaction components. Postrun software (LCMS Postrun version 2.02, Shimadzu Scientific Instruments, Columbia, MD) was used to analyze the data from the ESI-MS chromatogram. Peaks were integrated to determine the relative intensity of each ion species monitored as compared to an internal standard.

Enzyme Activity Determination. The enzymatic activity determination was as previously reported. ${ }^{1}$ One unit of enzyme is defined as the amount of enzyme required to consume one micromole $\alpha$-D-glucose-1-phosphate (Glc-1-phosphate) per minute.

\footnotetext{
${ }^{1}$ Zea, C. J.; Pohl, N. L. Anal. Biochem. 2004, 328, 196-202.

${ }^{2}$ Jiang, J.; Biggins, J. B.; Thorson, J. S. J. Am. Chem. Soc. 2000, 122, 6803-6804.
} 


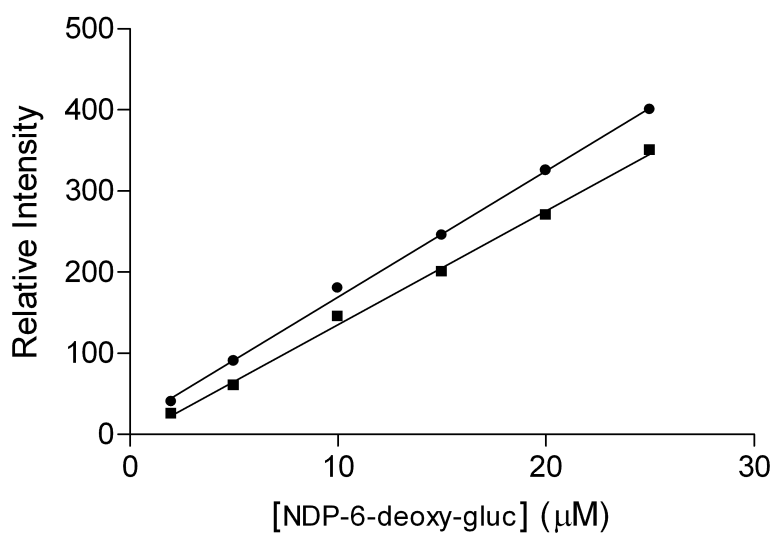

FIGURE S3. Calibration curve for UDP-6dGlc (circle) and dTDP-6dGlc (square).

Standard Curves. Calibration curves for UDP-Glc and dTDP-Glc were constructed as previously reported. ${ }^{1}$ Calibration curves of UDP-6dGlc and dTDP-6dGlc were created by allowing the E. coli UDP-glucose pyrophosphorylase enzyme to react with 6dGlc-1phosphate $(500 \mu \mathrm{M})$ in the presence of a limiting amount of UTP and dTTP $(2-25 \mu \mathrm{M})$ in a reaction mixture containing tris(hydroxymethyl)aminomethane (Tris) buffer $(25 \mathrm{mM}$, $\mathrm{pH} 7.8$ ), inorganic pyrophosphorylase (IPP, $0.2 \mathrm{U}$ ), and UDP-glucose pyrophosphorylase $(0.2 \mathrm{U})$. Reactions were carried out at $37^{\circ} \mathrm{C}$ and after $1 \mathrm{~h}$, as determined by the complete disappearance of UTP or dTTP by ESI-MS, $30 \mu \mathrm{L}$ of the reaction mixture was quenched by addition to $30 \mu \mathrm{L}$ of $70 \%$ methanol/water containing adenosine 5'-monophosphate (AMP, $6 \mu \mathrm{M}$ ) as an internal standard. The quenched solutions were centrifuged 10 minutes at $10,000 \times \mathrm{g}$ to precipitate the protein. Aliquots $(15 \mu \mathrm{L})$ of the reaction mixtures were diluted with $135 \mu \mathrm{L}$ of acetonitrile/water/triethylamine (35/65/0.2). These samples $(30 \mu \mathrm{L})$ were subjected to analysis via ESI-MS to determine the amount of UDP-6dGlc $(m / z=549)$ and dTDP-6dGlc $(m / z=547)$ produced as compared to the internal standard, AMP $(m / z=346)$. The relative intensity of the product was plotted against the concentration of UTP or dTTP. It was found that the ionization of UDP-6dGlc was the same for UDP-4dGlc and UDP-3dGlc; therefore, the calibration curve for UDP-6dGlc was used to determine the kinetic values for UDP-4dGlc and UDP-3dGlc.

Relative acceptance of deoxyglucose-1-phosphates. The relative acceptance rates of the deoxyglucose-1-phosphates were determined from the formation of UDP-dGlc and dTDP-dGlc using ESI-MS and compared to the acceptance of the natural substrates UTP and Glc-1-phosphate. The reaction was initialized by the addition of Glc-1-phosphate or dGlc-1-phosphate ( $5 \mathrm{mM} \alpha$ anomer) to a reaction mixture containing Tris buffer $(25 \mathrm{mM}$, $\mathrm{pH} 7.6$ or 7.8), IPP (0.2 U), UDP-glucose pyrophosphorylase (0.02 U), and UTP or dTTP $(10 \mu \mathrm{M})$. Reactions were carried out at $37^{\circ} \mathrm{C}$ and after 30 minutes $15 \mu \mathrm{L}$ of the reaction mixture was quenched by addition to $30 \mu \mathrm{L}$ of $70 \%$ methanol/water containing AMP (3 $\mathrm{mM})$ as an internal standard. The quenched solutions were centrifuged 10 minutes at $10,000 \times \mathrm{g}$ to precipitate the protein. Aliquots $(15 \mu \mathrm{L})$ of the reaction mixtures were diluted with $135 \mu \mathrm{L}$ of acetonitrile/water/triethylamine (35/65/0.2). These samples $(3 \mu \mathrm{L})$ were subjected to analysis via ESI-MS to determine the amount of UDP-6dGlc, dTDP6dGlc, UDP-4dGlc, or UDP-3dGlc formed. 
Measurement of $K_{M}$ and $V_{\max }$ of dGlc-1-phosphates. The values of $K_{M}$ and $V_{\max }$ were derived from enzymatic reactions run in triplicate and determined from the initial rates of UDP-6dGlc, dTDP-6dGlc, UDP-4dGlc, or UDP-3dGlc formation by monitoring the rate of UDP-6dGlc, dTDP-6dGlc, UDP-4dGlc, or UDP-3dGlc formation using ESI-MS. The reaction conditions used were taken from a previously reported procedure. ${ }^{1}$ The enzymatic reaction was initiated by the addition of the dGlc $(2-100 \mu \mathrm{M})$ to a reaction mixture containing Tris buffer $(25 \mathrm{mM}$, pH 7.6 or 7.8$)$, IPP (0.2 U), UDP-glucose pyrophosphorylase $\left(2 \times 10^{-5} \mathrm{U}\right)$, and UTP or dTTP $(300 \mu \mathrm{M})$. Reactions were carried out at $37{ }^{\circ} \mathrm{C}$ and after 5 minutes $30 \mu \mathrm{L}$ of the reaction mixture was quenched by addition to $30 \mu \mathrm{L}$ of $70 \%$ methanol/water containing AMP $(6 \mu \mathrm{M})$ as an internal standard. The quenched solutions were centrifuged $10 \mathrm{~min}$ at $10,000 \times \mathrm{g}$ to precipitate the protein. Aliquots $(15 \mu \mathrm{L})$ of the reaction mixtures were diluted with $135 \mu \mathrm{L}$ of acetonitrile/water/triethylamine $(35 / 65 / 0.2)$. These samples $(30 \mu \mathrm{L})$ were subjected to analysis via ESI-MS to determine the amount of UDP-6dGlc, dTDP-6dGlc, UDP-4dGlc, or UDP-3dGlc formed. Anomeric mixtures of phosphates could be used as reported with another sugar nucleotidyltransferase ${ }^{2}$ with concentration values calculated for only the $\alpha$ anomer present. 